\title{
EVIDÊNCIAS PRELIMINARES DA ATENÇÃO À SAÚDE DAS PESSOAS PORTADORAS DE DEFICIÊNCIA NAS UNIDADES BÁSICAS DE SAÚDE EM UMA CIDADE DO ESTADO DO PARANÁ, BRASIL
}

\author{
PRELIMINARY EVIDENCE OF HEALTH CARE FOR PEOPLE WITH DISABILITIES IN THE BASIC \\ HEALTH UNITS OF A CITY IN THE STATE OF PARANÁ, BRAZIL
}

\section{Alessandra Hilgemberga, Juliana Regina Dias Lemos ${ }^{b}$, José Rosa Gomes}

\author{
aahilgemberg@hotmail.com, blemos.jrd@terra.com.br, c1967jrgomes@gmail.com
} Universidade Estadual de Ponta Grossa - Ponta Grossa (PR), Brasil

Data de recebimento do artigo: 19/07/2017 Data de aceite do artigo: 25/10/2017

\section{RESUMO}

No Brasil, a implementação de políticas de saúde pública de atenção às pessoas com deficiência física ainda é um objetivo a ser alcançado, apesar de existir uma legislação pertinente que garante a atenção integral para essa fração da população. Portanto, cabe a todas as esferas do governo assegurar e avaliar a implementação dessas açôes através do financiamento e da formação dos profissionais de saúde, sejam médicos, técnicos ou profissionais de enfermagem. É necessário também que pesquisas acadêmicas contribuam para a avaliação sistemática da implementação dessas políticas. Dessa forma, esta investigação foi realizada no sentido de apresentar evidências preliminares da atenção à saúde da pessoa portadora de deficiência (PPD) nas 46 Unidades Básicas de Saúde (UBS) da zona urbana de uma cidade do estado do Paraná, Brasil, no período de 2016. O método utilizado foi exploratório, através da aplicação de um questionário direcionado ao responsável pela UBS, que abordava os seguintes aspectos: (i) formação dos profissionais de saúde para atenção a PPD; (ii) infraestrutura (arquitetônica e de condiçóes de trabalho); e (iii) perfil da PPD atendida nas UBS. Nesta pesquisa, apenas 36 questionários foram respondidos, e os resultados apontaram que: (i) há pouca intimidade dos profissionais nas UBS com a população de PPD atendida, indicando falta de formação na graduação, falta de formação da equipe de apoio, bem como falta de programas de saúde direcionados; (ii) as UBS não possuem condições de infraestrutura, sejam arquitetônicas ou de material de apoio, voltadas para a atenção das PPD; e (iii) nenhuma UBS possui programas de saúde voltados para as PPD. Conclui-se que existem fragilidades em relaçâo a todos os pontos avaliados nesta pesquisa, no que se refere à atenção à saúde da PPD nas UBS já determinadas em lei.

Palavras-chave: Centros de saúde; atenção à saúde; Sistema Único de Saúde.

\section{ABSTRACT}

In Brazil, the implementation of public health policies for the care of people with physical disabilities is still an objective to be achieved, since there is a pertinent legislation that guarantees comprehensive care for this fraction of the population. It is incumbent upon all government spheres to ensure and evaluate the implementation of these actions through financing and training of health professionals such as doctors, technicians or nursing professionals. It is also necessary that academic research contribute to the systematic evaluation of these policies implementation. Thus, this investigation was carried out to present preliminary evidence about health care of people with disabilities (PWD) in the 46 Basic Health Units (BHU's) of a city's urban area in the state of Paraná, Brazil, in 2016. The exploratory method was adopted, by using a questionnaire directed to the head of each BHU addressing the following aspects: (i) training of health professionals for PWD care; (ii) infrastructure (architectural and working conditions); and (iii) profile of PWD assisted in the BHU. In this research, only 36 questionnaires were answered, and the results showed that: (i) there is little intimacy among the professionals in the BHU and the PWD population assisted, which indicates lack of training at graduation, of the support team, as well as lack of 
targeted health programs; (ii) the BHU's do not have infrastructural conditions, whether architectural or support material, focused on PWD attention; (iii) none of the evaluated BHU's has health programs focused on PWD. The conclusion is that there are weaknesses related to all the points evaluated in this research, regarding the health care of PWD in BHS's in compliance with the determinations of the Brazilian law.

Keywords: Health centers; health care; Unified Health System.

\section{Introdução}

Conforme definido no artigo $4^{\circ}$ da Lei 8.080/1990, o Sistema Único de Saúde (SUS) é o conjunto de açōes e serviços de saúde prestados por órgãos e instituições públicas federais, estaduais e municipais, da Administração Direta e Indireta e das fundações mantidas pelo poder público e, complementarmente, pela iniciativa priva$\mathrm{da}^{1}$. O SUS foi criado, a partir das manifestaçōes de um conjunto de necessidades sociais de saúde, as quais imprimem um caráter ético-moral defendidas como direito de todo cidadão. Enquanto conquista das lutas participativas e democráticas, o SUS se desenvolve com base nos princípios de acesso, universalidade, equidade e integralidade, e com base nas diretrizes organizativas de descentralização, regionalização, hierarquização e participaçáo da comunidade ${ }^{2}$.

Entretanto, as questóes relativas ao acesso aos serviços de saúde sáo marcadas, de uma maneira geral, por situaçóes conflituosas que angustiam trabalhadores, gestores e usuários. A realidade aponta uma dificuldade ainda maior de atendimento para as pessoas portadoras de deficiência $(\mathrm{PPD})^{3}$. Segundo dados recentes do Censo do Instituto Brasileiro de Geografia e Estatística, há no Brasil cerca de 45,6 milhóes de pessoas com deficiência, o que corresponde a $23,92 \%$ da população brasileira. Observa-se, pois, que se trata de uma grande parcela da população, sendo boa parte dela usuária do SUS, e, como tal, deve ser assistida em todas as suas necessidades de saúde ${ }^{4}$. Para esse tipo de população, o Ministério da Saúde (MS), mediante uma série de documentos, define as estratégias de ação para a atenção aos portadores de deficiência (PD). A Política Nacional de Saúde da Pessoa com Deficiência possui, como propósitos gerais, um amplo leque de açóes que vai desde a proteção e prevençáo de agravos da saúde, passando pela reabilitação e inclusão da pessoa com deficiência em todas as esferas da vida social. Uma das diretrizes da política visa ao fortalecimento dos movimentos institucionais voltados à busca contínua da ampliação da acessibilidade e da inclusão, assim como à melhoria paulatina do acesso às estruturas físicas, às informaçôes e aos bens e serviços disponíveis aos usuários com deficiência no SUS4. Porém, foi apenas em 2015 que o governo sancionou a Lei 13.146, de 6 de julho de 2015, a qual considera pessoa com deficiência aquela que tem impedimento de longo prazo de natureza física, mental, intelectual ou sensorial, o qual, em interação com uma ou mais barreiras de infraestrutura, pode obstruir sua participação plena na sociedade em igualdade de condiçôes com as demais pessoas. Elenca também uma série de garantias de atenção integral à saúde em todos os níveis de complexidade, por intermédio do SUS, garantido acesso universal e igualitário, desde a atenção a partir das Unidades de Saúde da Família até os Serviços de Reabilitação, tratamento odontológico, procedimentos de enfermagem, acesso a medicamentos entre outros ${ }^{5}$.

No que compete aos profissionais de enfermagem em relação aos cuidados e assistência às pessoas com deficiência, enumeram-se atividades de autocuidado, tais como: higiene, nutrição, eliminação, atividades de vida diária associadas ao lar, cuidados à criança, entre outras. Neste contexto, é relevante destacar pesquisas em enfermagem cujo objeto de estudo aborda as características da comunicação entre as pessoas com deficiência sensorial e enfermeiros, com vistas a aprimorar a prática assistencial, mediante a construção de modelos de comunicação verbal e não verbal. Além disso, são diversas as possibilidades de atividades de educação e promoção da saúde junto com esta clientela, nas mais variadas temáticas ${ }^{6}$.

Assim, com o propósito de aprimorar a atenção à saúde dos portadores de deficiências, é importante o estudo e a avaliação contínua da formação dos profissionais de saúde, da infraestrutura das unidades de saúde e dos serviços prestados, a fim de efetivar e consolidar as práticas e açôes colocadas em lei. Aponta-se que as legislações voltadas para o direito da pessoa com deficiência, apesar de bem elaboradas e abrangentes, ainda precisam ser mais bem implementadas e fiscalizadas, para que se possa construir açóes facilitadoras da acessibilidade, possibilitando a inclusão social. Particularmente, no que se refere ao âmbito da saúde pública, observa-se que a assistência a essa população ainda apresenta um perfil de fragilidade, desarticulaçáo e descontinuidade de açóes, inserindo-a marginalmente no sistema de saúde.

Neste contexto, avaliar as açóes da atenção à saúde da pessoa com deficiência nas UBS de cada município se faz importante, no sentido de contribuir com o aperfeiçoamento dessas ações, dentro das propostas do SUS. Dessa forma, o objetivo desta pesquisa foi trazer evidências preliminares sobre a atenção à saúde das pessoas portadoras de deficiência (PPD) nas Unidades Básica de Saúde na região urbana da cidade de Ponta Grossa/PR, 
que se localiza no Sul do Brasil. Os objetivos específicos foram: (i) identificar o nível de conhecimento e formaçáo dos profissionais de saúde (enfermeiros e equipe de apoio) das UBS, para atender às PPD; (ii) avaliar as condiçóes de infraestrutura das UBS no tocante à acessibilidade e ao material de apoio à prática de atenção à saúde da PPD e (iii) identificar as ações, os programas, os serviços prestados e as dificuldades na prática da atenção de saúde às PPD, nas UBS.

\section{Metodologia}

A pesquisa foi exploratória, com abordagem quantitativa. Foram utilizados como premissas as seguintes definiçōes teóricas: (i) Barreira: qualquer entrave que limite ou impeça o acesso, a liberdade de movimento e a circulação com segurança das pessoas; (ii) Acessibilidade: possibilidade e condição de alcance para a utilização com segurança e autonomia dos espaços e equipamentos; (iii) Atenção à saúde: formação dos profissionais de enfermagem, o material necessário para uma atençáo efetiva e o conhecimento dos enfermeiros da legislação sobre a atenção à saúde dos portadores de deficiência.

\section{Procedimentos prévios à coleta dos dados}

O trabalho foi submetido à análise e autorização da Prefeitura da Cidade de Ponta Grossa bem como submetido à análise do Comitê de Ética em Pesquisa com Seres Humanos da Universidade Estadual de Ponta Grossa (Processo 1.667.457).

\section{Instrumento de coleta das informações}

Foi utilizado um questionário construído pelos pesquisadores, contendo dez questóes, o qual foi aplicado pela primeira vez neste estudo e pode ser encontrado no material suplementar. As questôes foram distribuídas em três partes. A primeira se refere à formaçáo dos profissionais que atuavam nas UBS: (i) o tempo de profissão, (ii) a capacitação para atenção ao portador de deficiência, (iii) autoavaliação da segurança na atençáo às PPDF (Pessoas Portadoras de Deficiência Física), (iv) avaliação da equipe de trabalho na UBS, (v) o conhecimento sobre a legislação referente à atenção às PPDF e existência de programas direcionados para a atenção dos PDF (Portador de Deficiência Física) e (vi) os fatores que interferem na atividade de atenção à PPDF. A segunda parte era sobre a infraestrutura: (i) a arquitetura da UBS e (ii) as barreiras de infraestrutura ainda presentes na unidade. Por fim, a terceira parte buscou traçar o perfil relacionado: (i) ao tipo de comunicação utilizada com o PDF e (ii) ao tipo de PDF que busca o serviço na UBS. Todo instrumento de avaliação (questionário) foi acompanhado com um termo de consentimento livre e esclarecido sobre os objetivos da investigação.

\section{Tamanho da amostra}

Nesta pesquisa apenas os(as) enfermeiros(as) que se declararam responsáveis pelas 43 UBS da zona urbana $\mathrm{da}$ cidade foram convidados a responder às questóes.

\section{Análise dos resultados}

Para análise dos resultados, as respostas para cada questão formulada, dentre os três temas abordados, foram tabuladas e apresentadas nas Figuras 1, 2 e 3, e expressas em números absolutos.

\section{Resultados}

Das 46 unidades básicas de saúde previstas para o estudo, 43 estavam em funcionamento. Deste total, apenas 36 enfermeiros preencheram o instrumento da pesquisa.

A Figura 1 e as letras de $\mathrm{A}$ a $\mathrm{F}$ correspondem às questóes referentes à primeira parte do questionário de avaliação. Em $1 \mathrm{~A}$ os resultados mostram que a maioria dos profissionais de enfermagem trabalham na UBS entre 1 e 3 anos. A Figura 1B mostra que a maioria dos profissionais não teve nenhum tipo de capacitação ou aperfeiçoamento, seja no ensino superior ou após a conclusão deste. Em 1C e 1D os resultados demostram que a equipe de trabalho nas UBS também não possui formação apropriada para atender com segurança às demandas de pacientes portadores de deficiência que procuram a UBS. Os resultados de 1C e 1D são confirmados pela Figura 1E, a qual mostra o desconhecimento, para a maioria dos profissionais, da legislação que norteia a atenção da PPD. A Figura 1E corrobora os resultados dessa primeira parte dos questionamentos mostrando que existem fatores que interferem na atenção eficiente a esse tipo de paciente, sendo destacado a falta de treinamento e a formação entre as opçóes apresentadas para essa questáo. Além disso, em função dos resultados apresentados de C até F, todas as UBS investigadas informaram que não desenvolvem nenhum programa específico de atenção à saúde da PPD. 
Figura 1: Resultados referentes à formação para atenção à PPD
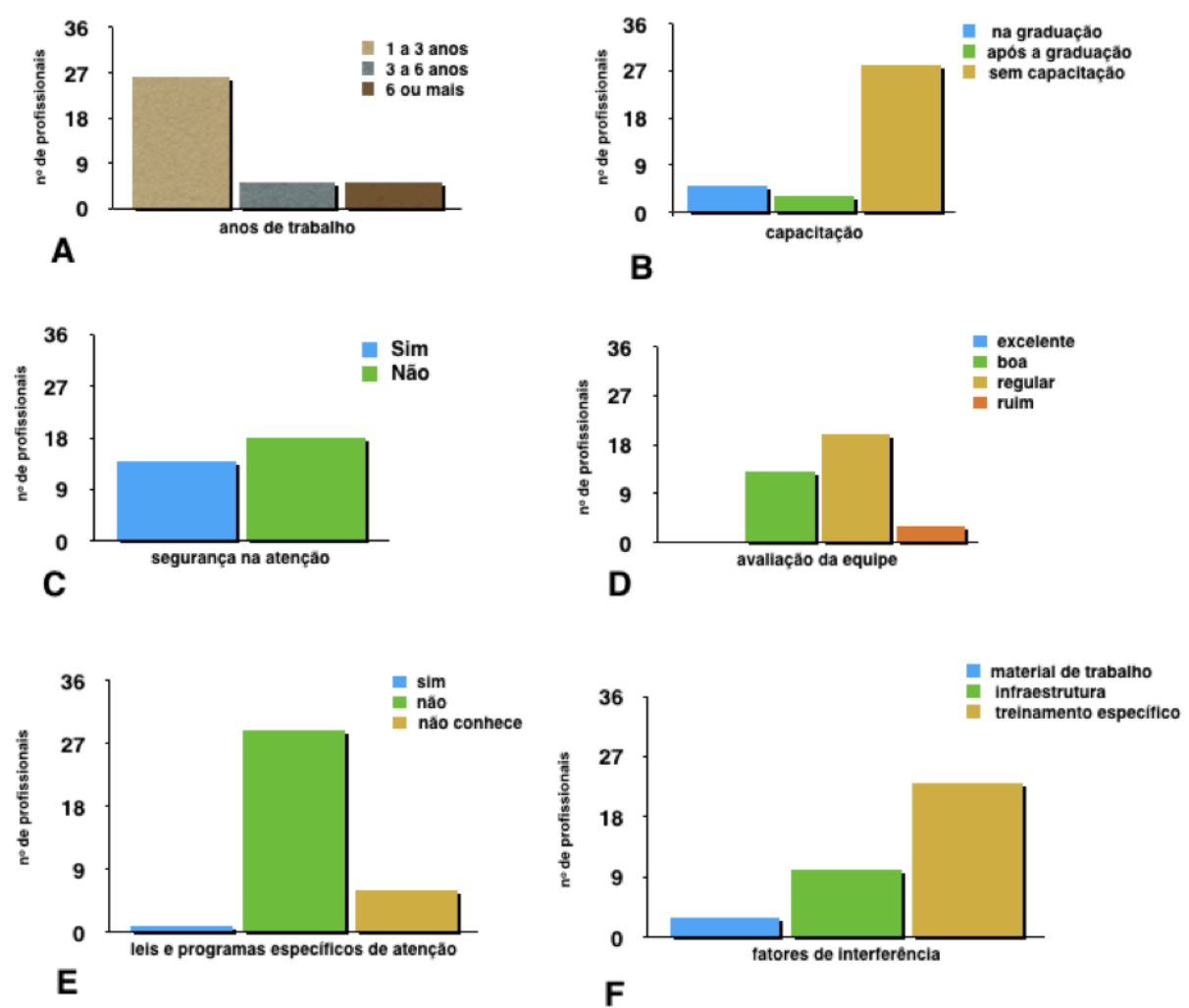

Fonte: Elaborado pelos autores, 2018.

Legenda: (A) Tempo de trabalho dos profissionais de enfermagem nas UBS do município; (B) Número de profissionais de enfermagem que tiveram treinamento específico para atenção à pessoa portadora de deficiência; (C) Número de profissionais de enfermagem que se sentem seguros. (D) Avaliação da equipe em relaçấo à atençáo à pessoa portadora de deficiência; (E) Número de profissionais de enfermagem que possuem conhecimento da legislação pertinente à atençáo da PPD; (F) Indicação dos fatores que interferem na efetivaçâo dos programas de saúde voltados para a PPD nas UBS avaliadas. (n=36).

A Figura 2 mostra os resultados da segunda parte avaliativa do questionário. Em $2 \mathrm{~A}$ pode ser observado que na maioria das UBS não existe infraestrutura adequada para a atenção à PPD e $2 \mathrm{~B}$ aponta que, dentre as deficiências da infraestrutura, se destacam a falta de calçadas adequadas para o acesso nas UBS, a falta de corrimão, banheiros e salas adaptadas para a atenção à PPD nas dependências da UBS entre outras como falta de rampas de acesso, cadeiras de roda e macas que facilitariam a atenção do profissional de saúde para a PPD.

Figura 2: Resultados referentes à infraestrutura da UBS para a atenção à PPD
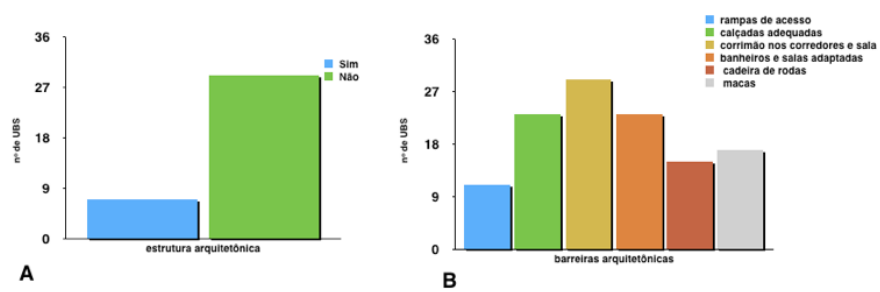

Fonte: Elaborado pelos autores, 2018.

Legenda: (A) Número de UBS que não possuem estrutura adequada ou adaptada para a atenção às pessoas portadoras de deficiência; (B) Principais barreiras encontradas para atenção às PPD nas UBS avaliadas. $(\mathrm{n}=36)$. 
A Figura 3 traça o perfil da forma como os profissionais se comunicam com as PPD, sendo a mais comum a verbal $(3 \mathrm{~A})$ e o tipo mais frequente de deficiente atendido nas UBS são os que possuem redução de mobilidade e os portadores de deficiência mental ou cognitiva (3B).

Figura 3: Resultados referentes à forma de comunicação dos profissionais com as PPD

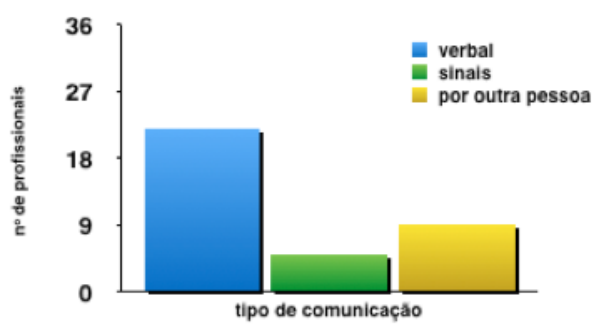

A

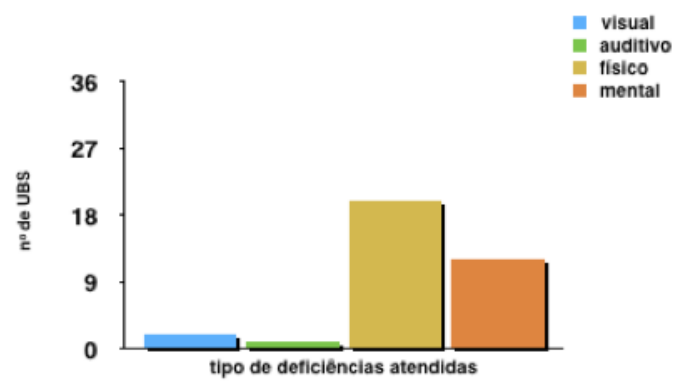

B

Fonte: Elaborado pelos autores, 2018.

Legenda: (A) Tipo de comunicação empregada pelos profissionais de enfermagem nas UBS durante a atenção às PPD; (B) Perfil das pessoas com deficiência atendidas nas UBS avaliadas. $(\mathrm{n}=36)$.

\section{Discussão}

Sabe-se que atenção primária deve viabilizar cuidados de promoção da saúde, prevenção, tratamento e reabilitação de doenças e agravos. No SUS, a UBS representa a porta de entrada para essa atenção primária e a partir dela, para serviços de saúde mais complexos. Dessa forma, a UBS deve se constituir como o local de excelência ao atendimento à populaçáo em geral, dada sua proximidade com a comunidade ${ }^{7}$. Segundo a Política Nacional de Atenção Básica ${ }^{8}$, é de responsabilidade da Estratégia Saúde da Família (ESF) a garantia do acesso universal aos serviços de saúde.

No Brasil, a Coordenação Geral de Saúde da Pessoa com Deficiência do Ministério da Saúde tem como propósitos gerais as seguintes normativas: proteger a saúde e reabilitar as pessoas com deficiência na capacidade funcional e desempenho (reabilitação física, auditiva e visual), garantir atendimento a pacientes com bolsas de ostomias e deficiência, vida social e prevenir agravos que determinem o aparecimento de deficiências. $\mathrm{O}$ principal objetivo desta Coordenação Geral é proporcionar atençâo integral à saúde da pessoa com deficiência, desde a atenção básica até a reabilitação, incluindo providenciar gratuitamente órteses, próteses e meios auxiliares de locomoção quando se fizerem necessário. Entretanto, todos esses propósitos para as pessoas que apresentam alguma deficiência náo acontecem sem um investimento adequado em infraestrutura e formaçáo especializada ${ }^{9,10}$.

Nesse contexto, os resultados desta pesquisa indicaram que a maioria dos profissionais de enfermagem se encontra no tempo de trabalho entre 1 e 3 anos na UBS. Se o tempo de trabalho na UBS pode ser um indicador da experiência com a atenção à saúde da populaçáo e, consequentemente, de que uma maior proximidade dos problemas existentes da comunidade afeta cada Unidade Básica de Saúde, os resultados indicam que a intimidade dos profissionais ainda é precoce e merece atenção para que seja mais efetiva no tocante à atenção a PPD.

Como o objetivo geral desta investigação foi o de apresentar evidências preliminares sobre a atenção a PPD nas UBS, verifica-se claramente que existe uma falha na formação do profissional atuante bem como da sua equipe. Dos 36 profissionais das UBS, apenas sete tiveram algum tipo de capacitação, durante a sua formação, para a atenção à pessoa portadora de deficiência. Da mesma forma, essa fragilidade na formação é evidenciada em outras questóes investigadas, como a da comunicaçáo com o paciente e a da segurança e treinamento do profissional e da sua equipe.

De forma geral, esses resultados evidenciam a falta de capacitação durante a formação e atualizaçăo, tanto dos profissionais de enfermagem quanto da equipe técnica, no manejo da atenção ao portador de deficiência. Neste ponto, esses resultados apontam a necessidade de uma revisão nos projetos pedagógicos dos cursos de formaçāo 
dos profissionais da área da saúde tanto em nível superior quanto em nível técnico, bem como nos cursos de pós-formação. É imprescindível que sejam priorizados conteúdos que abordem de forma prática as principais comorbidades dos portadores de deficiência para uma melhor formação dos profissionais da saúde. Dentre esses conteúdos, é importante se introduzir conceitos que abordem a Teoria da Enfermagem Transcultural, que se refere ao cuidado com a formaçáo do profissional no tocante à sensibilização da atenção pela comunicação adequada com o paciente ${ }^{11}$. Um avanço no processo da comunicação entre profissionais e clientela tem ocorrido nos currículos de formação com a introdução do ensino de libras preconizado, entre outras orientaçôes, pela Secretaria Nacional das Pessoas com Deficiência dentro da formação e pós-formação de todos os profissionais da área de saúde em todos os níveis, desde os auxiliares até os que exercem a medicina.

Nos programas voltados para a atenção às PPD se preconiza que na estratégia do Programa Saúde da Família (ESF) exista a implantação de equipes matriciais de atendimento domiciliar de reabilitação na atenção básica, na qual para cada cinco equipes deve existir uma equipe de reabilitaçáo, composta por psicólogo, assistente social, fonoaudióloga, terapeuta ocupacional, fisioterapeuta, médico, enfermeiro, nutricionista, técnico de enfermagem, pedagogo, intérprete de libras, dentre outros $^{4}$. Entretanto, é necessário que essas ações sejam permanentemente avaliadas na sua implantação e eficiência de execução. De fato, é fundamental que exista uma formação adequada dos profissionais para a execução adequada da atenção aos portadores de deficiência, ficando evidente em nossa investigação preliminar a falha das instituições de formação tanto em nível superior quanto de nível técnico. No estado do Paraná foi idealizado o Plano Estadual da Rede de Cuidados à Pessoas com Deficiência (PERCPD) ${ }^{12}$. Dentre as diversas redes propostas neste plano, está a Rede de Cuidados à Pessoa com Deficiência que é composta por três grandes níveis: 1) Atenção primária, 2) Atenção Especializada em Reabilitação e 3) Atenção hospitalar e de Urgência e Emergência.

No que se refere à Atenção Primária, os componentes da rede estão estruturados da seguinte forma: 1) Unidades de Atenção Básica e Estratégia de Saúde da Família; 2) Núcleo de Apoio à Saúde da Família (NASF), quando houver; e 3) Atenção em Saúde Bucal. Segundo o PERCPD, para a atenção primária, as açôes deverão ser desenvolvidas preferencialmente pelas Equipes de Saúde da Família com o suporte dos NASF onde houver, ou nas UBS.

Assim, considerando as UBS como as organizadoras da efetivação dessa rede de atenção às PPD, os resultados desta pesquisa indicam a inexistência nas UBS avaliadas de programas voltados aos deficientes. Neste contexto, apesar do objetivo estar direcionado para a atenção à saúde das PPD na UBS, ficou evidenciado a princípio que, mesmo com a existência de outras açóes como ESF ou NASF, há falha na implantação ou execução do PERCPD nessa cidade em nível de atenção primária. Ainda fica mais claro essa falha no PERCPD, quando sáo consideradas as causas da inexistência dessa atenção no município, merecendo como destaque a falta de treinamento específico, a falta de material de trabalho e a falta de infraestrutura.

Já em relação às questóes relacionadas com a parte da infraestrutura, que também compóem o PERCPD, os resultados mostraram que todas as barreiras apresentadas como opções no questionário ainda precisam ser vencidas nas UBS. Esses resultados contradizem o que determina a Associação Brasileira de Normas Técnicas $(\mathrm{ABNT})$, ao prever que os locais de utilização pública e privada, de uso comunitário, onde se incluem as UBS, devem ser adaptados, com o objetivo de promover a maior acessibilidade possível aos indivíduos, e que as rampas não podem ter pisos escorregadios e obrigatoriamente devem ter corrimãos, além de banheiros adapta$\operatorname{dos}^{13}$. A prevalência de barreiras arquitetônicas evidenciadas em nossa pesquisa nas UBS de Ponta Grossa são preocupantes, pois a maioria delas náo atendem às normatizaçóes para aqueles que necessitam de atenção especial, no sentido de garantir que eles possam recorrer a estes serviços quando necessário, com total autonomia, sem ter que enfrentar dificuldades ou solicitar ajuda de outras pessoas, o que pode ser referencial da qualidade da atenção à saúde ${ }^{13}$.

Diante dos resultados coletados nesta pesquisa, é evidente a existência de falhas nas ações da gestão em saúde para a atenção aos deficientes nas UBS da cidade avaliada, falhas estas que vão desde a formação do profissional, seja em nível superior ou técnico, passando pela infraestrutura e chegando nos serviços ou programas de saúde que deveriam ser direcionados às pessoas portadoras de deficiência, nas UBS.

Assim, considerando que a Unidade Básica de Saúde é a porta de entrada do cidadáo para os serviços de saúde e para outros níveis de maior complexidade dentro do sistema, é importante que os gestores criem condiçóes tanto de formaçáo como de infraestrutura para uma atenção de qualidade aos portadores de deficiência em cada UBS, ou que sejam criadas UBS voltadas para a atenção das PPD, com uma equipe multiprofissional treinada, além de toda a infraestrutura tanto de acessibilidade quanto de execução clínica, como salas de consultas e equipamentos de rotina para diagnóstico. Entretanto, essa realidade não é exclusiva da cidade 
avaliada, outros trabalhos também indicaram a existência do descompasso entre a proposta das políticas públicas no país e a realidade da atenção à saúde ${ }^{10}$. Espera-se que este trabalho preliminar contribua para a discussáo e as avaliaçóes futuras das demandas do tema e para a efetiva melhoria da Atenção Primária à Saúde das pessoas portadoras de deficiência na cidade de Ponta Grossa e nos municípios brasileiros.

\section{Conclusões}

Os resultados desta investigação permitiram concluir que existem fragilidades em relaçáo a todos as questóes propostas no questionário utilizado como instrumento de avaliação, no qual destacam-se a falta de formação dos profissionais e as falhas na infraestrutura das UBS para a atenção à saúde da pessoa portadora de deficiência nas Unidades Básica de Saúde da cidade de Ponta Grossa/PR.

\section{Referências}

1. Brasil. Lei no 8.080, de 19 de setembro de 1990. Lei Orgânica da Saúde. Diário Oficial da Uniāo [Internet]. 1990 set 20 [citado em 2017 mar 4];1:18055. Disponível em: https://goo.gl/TzkgK1.

2. Brasil. Constituição da República Federativa do Brasil. Diário Oficial da Uniāo [Internet]. 1988 [citado em 2018 mar 15]. Disponível em: https://goo.gl/8nJZ9a

3. Lopes MJM, Soares JSF, Bohusch G. Usuários portadores de deficiência: questôes para a atenção primária de saúde. Rev Baiana Enferm. 2014;28(1):4-12.

4. Brasil. Portaria no 1.060 , de 5 de junho de 2002. Política Nacional de Saúde da Pessoa Portadora de Deficiência.
Diário Oficial da União [Internet]. 2002 jun 10 [citado em 2017 mar 4];1:21. Disponível em: https://goo.gl/BFt78X.

5. Brasil. Lei no 13.146, de 6 de julho de 2015. Institui a Lei Brasileira de Inclusão da Pessoa com Deficiência (Estatuto da Pessoa com Deficiência). Diário Oficial da União [Internet]. 2015 jul 7 [citado em 2017 mar 4];1:2. Disponível em: https://goo.gl/BjyKrP.

6. Cunha ABO, Silva LMV. Acessibilidade aos serviços de saúde em um município do Estado da Bahia, Brasil, em gestão plena do sistema. Cad Saúde Pública. 2010;26(4):725-37.

7. Brasil. Ministério da Saúde. Secretaria de Atenção à Saúde. Departamento de Atenção Básica. Política Nacional de Atenção Básica. Brasília, DF: Ministério da Saúde; 2012.

8. Siqueira FCV, Facchini LA, Silveira DS, Piccini RX, Thumé E, Tomasi E. Barreiras arquitetônicas a idosos e portadores de deficiência física: um estudo epidemiológico da estrutura física das unidades básicas de saúde em sete estados do Brasil. Ciênc Saúde Colet. 2009;14(1):39-44.

9. Nogueira GC, Schoeller SD, Ramos FRS, Padilha MI, Brehmer LCF, Marques AMFB. Perfil das pessoas com deficiência física e Políticas Públicas: a distância entre intenções e gestos. Ciênc Saúde Colet. 2016;21(10):3131-42.

10. Queiroz MVO, Pagliuca LMF. Conceito de enfermagem transcultural: análise de seu desenvolvimento em uma dissertação de mestrado. R Bras Enferm. 2001;54(4):630-7.

11. Paraná (Estado). Secretaria da Saúde. Plano Estadual da Rede de Cuidados às Pessoas com Deficiência [Internet]. Paraná, 2015 [citado em 2017 mar 4]. Disponível em: https://goo.gl/T3KaQi.

12. Associação Brasileira de Normas Técnicas. ABNT/CB040 - Comitê Brasileiro de Acessibilidade [Internet]. São Paulo: ABNT [citado em 2016 dez 4]. Disponível em: https://goo.gl/c3Sj7u.

13. França ISX, Pagliuca LMF Acessibilidade das pessoas com deficiência ao SUS: fragmentos históricos e desafios atuais. Rev RENE. 2008;9(2):129-37.

\section{Como citar este artigo:}

Hilgemberg A, Lemos JRD, Gomes JR. Evidências preliminares da atenção à saúde das pessoas portadoras de deficiência (PPD) nas unidades básicas de saúde em uma cidade do estado do Paraná, Brasil. Rev. Aten. Saúde. 2018;16(55):57-63. 